\title{
news
}

\section{news news news news news news news news}

\section{Mental health consequences of war and civil conflict}

The present escalation of conflict around the world in which civilians are the principal victims is of grave concern, with conflict having serious mental health consequences both for the participants and those they involve, the World Federation for Mental Health (WFMH) has said in a statement

Promoting and protecting the mental health and the well-being of the population must be a high priority for governments, says the WFMH, specifically noting the circumstances in Gaza, Darfur, Sri Lanka, and the Congo, among other areas, and pointing out that it does not take sides in armed conflicts. Paying attention to the mental and emotional health of a nation's people must be given added priority during times of stress and conflict such as the world is currently experiencing

Tension, disruption and uncertainty caused by war and civil strife have major and lasting consequences in the daily lives and routines of children and adults in areas of ongoing violence. Anger and worries about terrorism and military conflicts, the very real fear for safety and survival of civilians and military personnel - all heighten stress and anxiety for everyone. For some, they bring strong feelings of hopelessness and depression and may result in post-traumatic stress, and they add further stress for those already living with a major illness. The overwhelming and constant connection to military and political conflicts created by the worldwide media lends a sense of 'virtual reality' to unfolding events that is inescapable.

Of major concern is that the mental health consequences of the hardship and trauma created by violence and disruption are generally being ignored by combatants on both sides of these conflicts, the WFMH says.

In the statement the WFMH issues an urgent call to nations involved in conflict, and to those contributing aid to support such conflict, to respond specifically to these issues and to consider the serious immediate and long-term mental health consequences of continuing armed conflict.

The international mental health community, including professional associations and NGOs concerned with health, is called upon to intervene with respective governments to insist on upholding the Geneva conventions concerned with the health consequences of war and to provide immediate financial support to the mental health services located in conflict areas.

In addition the major humanitarian organisations that traditionally have not prioritised mental health are called upon to provide the means to support established organisations like the WFMH to expand training and services according to the new InterAgency Standing Committee Guidelines on Mental Health and Psychosocial Support in Emergency Settings.

Source: www.wfmh.org

\section{World Mental Health Day 2009}

The campaign for the 2009 World Mental Health Day, which takes place on 10 October, will focus on 'Mental health in primary care: Enhancing treatment and promoting mental health'.

This year's theme will address the continuing need to make mental health issues a global priority, and will stress the all too often neglected fact that mental health is an integral element of every individual's overall health and well-being.

The campaign theme is intended to draw worldwide attention to the growing body of information and knowledge focusing on the integration of mental health in primary health care, and to provide this information to grassroots patient/consumer, family member/caregiver, and advocacy and educational mental health associations around the world. This is a significant trend in shifting mental health diagnosis, treatment and care from the traditional separate mental health services delivery system into mainstream health care.

World Mental Health Day 2009 will highlight the opportunities and the challenges that integrating mental health services into the primary health care delivery system will present to people living with mental disorders and poor mental health, to their families and caregivers as well as to health care professionals. As always, the campaign will focus on the critical role that mental health advocacy, patient/service user, and family/caregiver organisations need to play in shaping this major general health and mental health reform movement

Advocates, families, professionals and policymakers across the global mental health sector must remember that this current movement to improve the way in which mental health services are delivered is not the first such reform effort. Lessons learned from the past tell us that achieving parity in how mental health services are addressed in countries around the world is not an easy struggle. The effective integration of mental health into primary care at a level of priority appropriate to the documented burden of care of mental illnesses will be a major undertaking in a time of global economic and social difficulty.

Source: www.wfmh.org/09world_mental_health_day_2009 htm 